\title{
Wireless Adaptive Network Coding Strategy in Multiple-Access Relay Channels
}

\author{
Sha Wei*, Jun $\mathrm{Li}^{\ddagger}$, Wen Chen*广 and Hang $\mathrm{Su}^{*}$ \\ ${ }^{*}$ Department of Electronic Engineering, Shanghai Jiao Tong University, Shanghai, China, 200240 \\ $\dagger$ SKL for ISN, Xidian University, Xi' an, China \\ ${ }^{\ddagger}$ School of Electrical Engineering and Telecommunications, University of New South Wales, Sydney, NSW 2052, AUSTRALIA \\ Email: \{venessa724,wenchen, Hmilyanjohn\}@sjtu.edu.cn; jun.li@unsw.edu.au
}

\begin{abstract}
This paper considers a multiple-access relay channel (MARC) with two sources, one relay and one destination, where the relay decides what it transmits to the destination according to the outage condition of source-relay links. The outage probability and the approximate bit error rate (BER) are derived, whiche are shown to be in tight match with Monte-Carlo simulation results. Simulation results reveal that adaptive DF strategy yields better performance than the traditional fixed DF strategies.

Index Terms - adaptive, decode-and-forward strategy, BER, outage probability
\end{abstract}

\section{INTRODUCTION}

The multiple-access relay channels (MARC) system [1][2] is an important communication scenario and has been recognized as one of the fundamental building blocks for modern wireless networks. And the network coding principle at the intermediate relays could alleviate the loss in spectral efficiency for such multi-user cooperative system. In this case, the signal processing plays a significant role in determining the end-to-end performance of a wireless network consisting of multiple source nodes. However, any wrong decision made at early hops can be propagated to the later ones. Thus it will lead to a poor system performance.

Conventional network coding is performed on the assumption that the transmissions of the sources are not in outage and are correctly decoded at the relay, which may not be the case with poor quality source-relay channels. If at least one of the messages is incorrectly decoded, then the network-coded message does not help in recovering the sources' messages at the destination node. Although some adaptive DF strategies have been proposed in single-source relay system [3][4][5], there is no paper working on multiple-access relay channels with interference. In this case, we propose an adaptive decodeand-forward (DF) cooperation protocol such that the relay forwards the network-coded message only when both sourcerelay links are not in outage for MARC system. If this is not the case, the relay either forwards the non-outage link corresponding message, or remains silent. The outage probability is derived when the source-relay channels are nonideal. Since the two sources transmit their information to the relay and destination simultaneously in the first time slot, the relay and destination receive mutually interfered message. The outage events analysis is different and complicated than the orthogonal multiple-access channel [6].

To analyze the BER performance of the adaptive DF cooperation protocol, we adopt a new methodology where all the possible received signals both from two sources to destination in the first time slot, and from relay to destination in the second time slot are mapped onto the so-called effective instantaneous coordinate. The coordinate is built for the time orthogonality of two-time slot received signals. Because the system is perfectly synchronized and with coherent detection, we choose four reference points such that the X-label of the reference point is the noise-free signal from direct source-destination link, and the Y-label is the noise-free signal from relay-destination link. Later we define the decoding regions corresponding to each reference points. Then the BER is computed as the probability that the equivalent noise is out of scope of the correct decoding region according to the minimum Euclidean distance strategy. Based on the formed coordinate, reference points and decoding regions, we derive the BER performance of the adaptive DF cooperation protocol.

The superscript $*$ is used to represent the conjugation operation. $\mathbb{E}[x]$ and $|x|$ denote the standard expectation operator and the absolute value of $x$, respectively. $\mathcal{N}\left(\mu, \sigma^{2}\right)$ denotes Gaussian distribution with mean $\mu$ and variance $\sigma^{2}$. And $f\left(x, y ; \mu_{x}, \mu_{y}, \Sigma\right)$ denotes a bivariant two-dimensional Gaussian distribution $\frac{1}{2 \pi \sigma^{2}} \exp \left(-\frac{1}{2}\left[\frac{\left(x-\mu_{x}\right)^{2}}{\sigma^{2}}+\frac{\left(x-\mu_{x}\right)^{2}}{\sigma^{2}}\right]\right)$, in which two random variables $X$ and $Y$ are independent, with mean $\mu_{x}$ and $\mu_{y}$ respectively, and covariance $\Sigma=$ $\left[\sigma^{2}, 0 ; 0, \sigma^{2}\right] . I(2)$ is a $2 \times 2$ identity matrix and $H^{\dagger}$ is the conjugate transpose of matrix $H$.

\section{System Model}

We consider a multiple-access relay channel system with two-source, one-relay and one-destination, as Fig. 1 shows. The two sources $\mathcal{S}_{1}$ and $\mathcal{S}_{2}$ transmit their information to the common destination $\mathcal{D}$ with the assistance of a half-duplex relay node $\mathcal{R}$. The two sources are assumed to randomly locate in a circle around the relay with the angles $\varphi_{1}$ and $\varphi_{2}$ (uniformly distributed in $(0,2 \pi]$ ), respectively. The channel between any two given nodes $j$ and $k$ is denoted by $h_{j k}$, with a subscript indicating the nodes under consideration, e.g., $j, k=\{1,2, \mathcal{R}, \mathcal{D}\}, h_{j k} \sim \mathcal{C N}\left(0,1 / \lambda_{j k}\right)$ satisfies complex normal distribution with zero mean and variance $1 / \lambda_{j k}$. The 


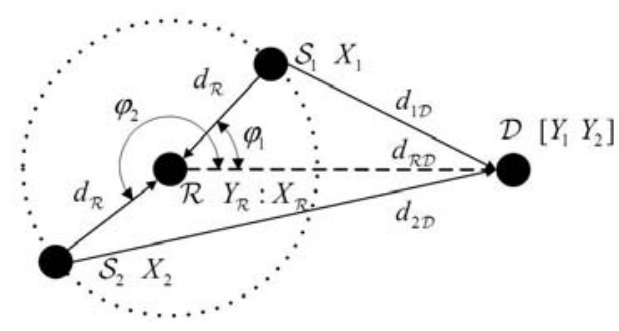

Fig. 1. The system model of the MARC with two-source, one-relay, and onedestination. The arrows with solid lines represent the first transmission phase and the arrow with dashed line represents the second transmission phase.

parameter $\lambda_{j k}$ is determined by the path loss model, and it is proportional to inter-node distance according to $\lambda_{j k} \propto\left(d_{j k}\right)^{\gamma}$, where $d_{j k}$ is the distance between the relevant nodes, and $\gamma$ is the attenuation exponent.

We split one transmission period into two phases. The first phase is composed of $t$ time slots, in which the two sources simultaneously broadcast their codewords $X_{1}$ and $X_{2}$, respectively to both the destination and the relay. The second phase is composed of $(1-t)$ time slots, in which the two sources keep silent while the relay processes the received signal and forwards the regenerated codeword $X_{\mathcal{R}}$ to the destination. After the second phase, the destination decodes the information of the sources by combining the received signals of the two phases.

We assume that the transmitted codewords $\mathbf{X}_{i}, i=1,2$ and $\mathbf{X}_{\mathcal{R}}$ are Binary Phase Shift Keying (BPSK) signals. We have $\mathbf{X}_{i}=\left[x_{i}^{1}, \cdots, x_{i}^{t L}\right]^{T}$ and $\mathbf{X}_{\mathcal{R}}=\left[x_{\mathcal{R}}^{1}, \cdots, x_{\mathcal{R}}^{(1-t) L}\right]^{T}$ with codeword length $L$. All the sources' symbols are identically independent distributed (i.i.d.) with probability $P\left(x_{i}^{j}\right)=0.5$. All the channels are slow-fading and the additive noises at receivers are complex Gaussian distributed noise with zero mean and variance $\sigma^{2}$. To normalize the transmission power, we assume that both sources and relay employ unit transmission power. We can write the received signals at the relay and destination in the first time slot as $\mathbf{Y}_{\mathcal{R}}=h_{1 \mathcal{R}} \mathbf{X}_{1}+h_{2 \mathcal{R}} \mathbf{X}_{2}+\mathbf{N}_{\mathcal{R}}$ and $\mathbf{Y}_{1}=h_{1 \mathcal{D}} \mathbf{X}_{1}+h_{2 \mathcal{D}} \mathbf{X}_{2}+\mathbf{N}_{1}$, respectively; then in the second time slot, the received signal at destination is $\mathbf{Y}_{2}=h_{\mathcal{R D}} \mathbf{X}_{\mathcal{R}}+\mathbf{N}_{2}$, where $\mathbf{N}_{\mathcal{R}}$ is the noise at the relay, $\mathbf{N}_{1}$ and $\mathbf{N}_{2}$ are the noise at destination in the first and second phase, respectively.

\section{The Adaptive Decode-And-Forward Scheme And OUtage Probability Analysis}

In this section, we propose the adaptive DF scheme based on the outage events of source-relay channels.

\section{A. Preliminary}

To simplify the outage probability representations in the following subsections, we first obtain the individual and common outage probabilities of $\mathcal{S}_{1}$ and $\mathcal{S}_{2}$ at relay in the first time slot. And we will give the method of calculating related mutual information with binary inputs.

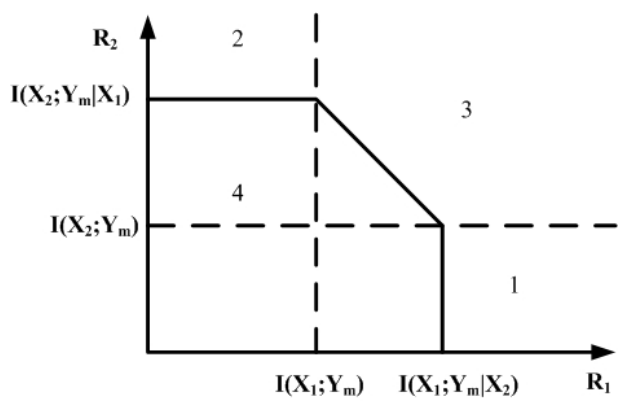

Fig. 2. Achievable rate region and outage regions for two-user MAC

In the first time slot, the source-relay channels form a twouser MAC system. Denote $R_{1}$ and $R_{2}$ be the transmission rates of channel $h_{1 \mathcal{R}}$ and $h_{2 \mathcal{R}}$ respectively, then the $\left(R_{1}, R_{2}\right)$ plane 2 is divided into four regions [7]. In region 1 , the message from $\mathcal{S}_{1}$ is decoded with error, while the message from $\mathcal{S}_{2}$ is decoded successfully. In this case, $R_{2}$ is less than the maximum rate when $X_{1}$ is regarded as Gaussian noise, i.e., $R_{2} \leq I\left(X_{2} ; Y_{\mathcal{R}} \mid h_{1 \mathcal{R}}, h_{2 \mathcal{R}}\right)$. Later we will omit the probability condition on channel state information $h_{1 \mathcal{R}}$ and $h_{2 \mathcal{R}}$ for notational simplicity. Likewise, in region 2 , the message $X_{2}$ is decoded with error, while the message $X_{1}$ is decoded successfully. In addition, region 3 denotes the area where decoding errors occur for both $X_{1}$ and $X_{2}$. And region 4 is the achievable rate region. Denote $P_{o, i}$ for $i=1, \cdots, 3$ as the outage probability of each region, and we assume $R_{1}=R_{2}=R$, then we have

$$
\begin{gathered}
P_{o, 1}=\operatorname{Pr}\left[I\left(X_{1} ; Y_{\mathcal{R}} \mid X_{2}\right)<R, I\left(X_{2} ; Y_{\mathcal{R}}\right) \geq R\right], \\
P_{o, 2}=\operatorname{Pr}\left[I\left(X_{1} ; Y_{\mathcal{R}}\right) \geq R, I\left(X_{2} ; Y_{\mathcal{R}} \mid X_{1}\right)<R\right], \\
P_{o, 3}=\operatorname{Pr}\left[I\left(X_{1} ; Y_{\mathcal{R}} \mid X_{2}\right)<R, I\left(X_{2} ; Y_{\mathcal{R}} \mid X_{1}\right)<R,\right. \\
\left.I\left(X_{1}, X_{2} ; Y_{\mathcal{R}}\right)<2 R\right],
\end{gathered}
$$

With the above setup, the individual outage probabilities that either $\mathcal{S}_{1}$ or $\mathcal{S}_{2}$ 's information is in outage, and the common outage probability that both $\mathcal{S}_{1}$ and $\mathcal{S}_{2}$ 's information are in outage in the first time slot are given by, respectively,

$$
\begin{gathered}
P_{o, \mathcal{S}_{1}}=P_{o, 1}+P_{o, 3}, \\
P_{o, \mathcal{S}_{2}}=P_{o, 2}+P_{o, 3}, \\
P_{o, \mathcal{S}_{1}, \mathcal{S}_{2}}=P_{o, 1}+P_{o, 2}+P_{o, 3} .
\end{gathered}
$$

Now, we show the method of calculating related mutual information with binary inputs. The conditional mutual information $I\left(X_{1} ; Y_{\mathcal{R}} \mid X_{2}\right), I\left(X_{2} ; Y_{\mathcal{R}} \mid X_{1}\right)$ and point-to-point mutual information $I\left(X_{\mathcal{R}} ; Y_{2}\right)$ can be calculated as that of the single link fading channels [8]. We will determine the mutual information $I\left(X_{1}, X_{2} ; Y_{\mathcal{R}}\right)$ and $I\left(X_{i} ; Y_{\mathcal{R}}\right)$ in the following.

We pick out the $j$-th symbol of $X_{i}$, i.e., $x_{i}^{j}$ and then we have $y_{\mathcal{R}}^{j}=h_{1 \mathcal{R}} x_{1}^{j}+h_{2 \mathcal{R}} x_{2}^{j}+n_{\mathcal{R}}^{j}$, where $y_{\mathcal{R}}^{j}$ and $n_{\mathcal{R}}^{j}$ are the $j$-th samples of $Y_{\mathcal{R}}$ and $N_{\mathcal{R}}$, respectively. We have $I\left(x_{1}^{j}, x_{2}^{j} ; y_{\mathcal{R}}^{j}\right)=\frac{1}{t L} I\left(X_{1}, X_{2} ; Y_{1}\right)$. The conditional probability function (PDF) belonging to $y_{\mathcal{R}}^{j}$ can be written as 
$p\left(y_{\mathcal{R}}^{j} \mid x_{1}^{j}, x_{2}^{j}\right)=\mathcal{N}\left(h_{1 \mathcal{R}} x_{1}^{j}+h_{2 \mathcal{R}} x_{2}^{j}, \sigma^{2}\right)$.

Since all the transmitted symbols are i.i.d., we have the probability $P\left(x_{1}^{j}=u, x_{2}^{j}=v\right)=P\left(x_{1}^{j}=u\right) P\left(x_{2}^{j}=v\right)=\frac{1}{4}$, $u, v \in\{-1,1\}$. Then $I\left(x_{1}^{j}, x_{2}^{j} ; y_{\mathcal{R}}^{j}\right)$ is calculated as (4).

Now we focus on the calculation of $I\left(x_{1}^{j} ; y_{\mathcal{R}}^{j}\right)=$ $\frac{1}{t L} I\left(X_{1} ; Y_{\mathcal{R}}\right)$. The related conditional PDF is written as

$$
\begin{aligned}
p\left(y_{\mathcal{R}}^{j} \mid x_{1}^{j}\right) & =\sum_{u=+1,-1} p\left(y_{\mathcal{R}}^{j} \mid x_{1}^{j}, x_{2}^{j}=u\right) P\left(x_{2}^{j}=u\right) \\
& =\frac{1}{2 \sqrt{2 \pi \sigma^{2}}} \exp \left\{-\frac{\left(y_{\mathcal{R}}^{j}-h_{1 \mathcal{R}} x_{1}^{j}-h_{2 \mathcal{R}}\right)^{2}}{2 \sigma^{2}}\right\} \\
& +\frac{1}{2 \sqrt{2 \pi \sigma^{2}}} \exp \left\{-\frac{\left(y_{\mathcal{R}}^{j}-h_{1 \mathcal{R}} x_{1}^{j}+h_{2 \mathcal{R}}\right)^{2}}{2 \sigma^{2}}\right\} .
\end{aligned}
$$

Then $I\left(x_{1}^{j} ; y_{\mathcal{R}}^{j}\right)$ is calculated as

$$
\begin{aligned}
I\left(x_{1}^{j} ; y_{\mathcal{R}}^{j}\right) & =\frac{1}{2} \sum_{u=-1,1} \int_{-\infty}^{\infty} p\left(y_{\mathcal{R}}^{j} \mid x_{1}^{j}=u\right) \\
& \cdot \log \left(\frac{2 p\left(y_{\mathcal{R}}^{j} \mid x_{1}^{j}=u\right)}{p\left(y_{\mathcal{R}}^{j} \mid x_{1}^{j}=1\right)+p\left(y_{\mathcal{R}}^{j} \mid x_{1}^{j}=-1\right)}\right) \mathrm{d} y_{\mathcal{R}}^{j} .
\end{aligned}
$$

\section{B. The Adaptive Decode-and-Forward Strategy}

In the proposed adaptive DF strategy, the relay either forwards the network-coded message, or forwards a single correctly decoded message, or remains silent depending on the outage events on source-relay channels. At the destination, identical messages are combined in a multiple-input multipleoutput (MIMO) manner. We assume each terminal transmits with rate $R$.

During the first time slot, source $\mathcal{S}_{1}$ broadcasts $X_{1}$ and $\mathcal{S}_{2}$ broadcasts $X_{2}$ concurrently to relay and destination. At relay $\mathcal{R}$, if the following three conditions are satisfied

$$
\begin{gathered}
I\left(X_{1} ; Y_{\mathcal{R}} \mid X_{2}\right)>R, \\
I\left(X_{2} ; Y_{\mathcal{R}} \mid X_{1}\right)>R, \\
I\left(X_{1}, X_{2} ; Y_{\mathcal{R}}\right)>2 R,
\end{gathered}
$$

the relay can correctly decode both sources using a maximum likelihood (ML) decoder [9]. If the relay correctly decode both $X_{1}$ and $X_{2}$, during the second time slot, $\mathcal{R}$ transmits message $X_{\mathcal{R}}=X_{1} \oplus X_{2}$ to $\mathcal{D}$. However, if the $h_{1 \mathcal{R}}$ link is not good enough such that (7) is not met but $I\left(X_{2} ; Y_{\mathcal{R}}\right)>R$ is satisfied, $\mathcal{R}$ can decode message $X_{2}$ by treating the signal transmitted by $\mathcal{S}_{1}$ as Gaussian noise. Similarly, if (8) is not met but $I\left(X_{1} ; Y_{\mathcal{R}}\right)>R, \mathcal{R}$ is capable of decoding $X_{1}$ by treating the signal transmitted by $\mathcal{S}_{2}$ as Gaussian noise. If the relay correctly decode either $X_{1}$ or $X_{2}$ but not both, $\mathcal{R}$ transmits message $X_{\mathcal{R}}=\hat{X}_{1}$ or $X_{\mathcal{R}}=\hat{X}_{2}$ to $\mathcal{D}$ using repetition codes during the second time slot. Otherwise, $\mathcal{R}$ can decode neither $X_{1}$ nor $X_{2}$, and remains silent in the second time slot.

Depending on the quality of the source-relay links, we classify the outage computation into four cases. Outage probabilities is computed in system level (i.e. considering the correct reception of both sources' packets at the destination). Denote
$\mathcal{E}_{(j, k)}$ be the event that a message of node $i$ transmitted in the link from node $j$ to node $k$ is correctly, and $\overline{\mathcal{E}}_{(j, k)}$ denotes the complementary outage event.

1) Case One: The relay decodes both sources' messages correctly, and forwards $X_{\mathcal{R}}=X_{1} \oplus X_{2}$ to $\mathcal{D}$. Let $\overline{\mathcal{E}}_{\mathcal{D}}$ be the outage event at destination after combining both the source and relay information, the system-level outage probability is

$$
P_{o}^{1}=\operatorname{Pr}\left(\mathcal{E}_{\left(\mathcal{S}_{1}, \mathcal{R}\right)} \bigcap \mathcal{E}_{\left(\mathcal{S}_{2}, \mathcal{R}\right)}, \overline{\mathcal{E}}_{\mathcal{D}}\right),
$$

where the superscript 1 (and later 2, 3, 4 in the following three cases) refers to the case number, $\operatorname{Pr}\left(\mathcal{E}_{\left(\mathcal{S}_{1}, \mathcal{R}\right)} \cap \mathcal{E}_{\left(\mathcal{S}_{2}, \mathcal{R}\right)}\right)=$ $P_{o, \mathcal{S}_{1}, \mathcal{S}_{2}}$ denotes the probability that relay can decode both $\mathcal{S}_{1}$ and $\mathcal{S}_{2}$ 's information correctly. In addition, $\operatorname{Pr}\left(\overline{\mathcal{E}}_{\mathcal{D}}\right)$ is determined by

$$
\operatorname{Pr}\left(\overline{\mathcal{E}}_{\mathcal{D}}\right)=\operatorname{Pr}\left(\log _{2}\left(\operatorname{det}\left(I(2)+\frac{H_{1} H_{1}^{\dagger}}{\Sigma}\right)\right)<2 R\right),
$$

where $H_{1}=\left[\begin{array}{ccc}h_{1 D} & h_{2 D} & 0 \\ 0 & 0 & h_{R D}\end{array}\right]$.

2) Case Two: The relay fails to decode message $X_{2}$, and correctly decodes $X_{1}$. Then relay forwards the repetition coded signal $\hat{X}_{1}$ to the destination in the second time slot. The outage probability is given by

$$
P_{o}^{2}=\operatorname{Pr}\left(\mathcal{E}_{\left(\mathcal{S}_{1}, \mathcal{R}\right)} \bigcap \overline{\mathcal{E}}_{\left(\mathcal{S}_{2}, \mathcal{R}\right)}, \overline{\mathcal{E}}_{\mathcal{D}}\right),
$$

where $\operatorname{Pr}\left(\mathcal{E}_{\left(\mathcal{S}_{1}, \mathcal{R}\right)} \cap \overline{\mathcal{E}}_{\left(\mathcal{S}_{2}, \mathcal{R}\right)}\right)=P_{o, 1}$ is the probability that relay can decode $X_{1}$ but not $X_{2}$, and $\operatorname{Pr}\left(\overline{\mathcal{E}}_{\mathcal{D}}\right)$ in Case 2 is calculated as

$$
\operatorname{Pr}\left(\overline{\mathcal{E}}_{\mathcal{D}}\right)=\operatorname{Pr}\left(\log _{2}\left(\operatorname{det}\left(I(2)+\frac{H_{2} H_{2}^{\dagger}}{\Sigma}\right)\right)<R\right),
$$

where $H_{2}=\left[\begin{array}{cc}h_{1 D} & h_{2 D} \\ h_{R D} & 0\end{array}\right]$.

3) Case Three: The relay fails to decode source one's message $X_{1}$, and correctly decode source two's message $X_{2}$. Thus, in the second time slot the relay forwards the regenerated signal $\hat{X}_{2}$ to the destination. The outage probability is

$$
P_{o}^{3}=\operatorname{Pr}\left(\overline{\mathcal{E}}_{\left(\mathcal{S}_{1}, \mathcal{R}\right)} \bigcap \mathcal{E}_{\left(\mathcal{S}_{2}, \mathcal{R}\right)}, \overline{\mathcal{E}}_{\mathcal{D}}\right),
$$

where $\operatorname{Pr}\left(\overline{\mathcal{E}}_{\left(\mathcal{S}_{1}, \mathcal{R}\right)} \cap \mathcal{E}_{\left(\mathcal{S}_{2}, \mathcal{R}\right)}\right)=P_{o, 2}$ and $\operatorname{Pr}\left(\overline{\mathcal{E}}_{\mathcal{D}}\right)$ are obtained similarly as case two by exchanging the position of $\mathrm{H}_{2}(2,1)$ and $\mathrm{H}_{2}(2,2)$ in matrix $\mathrm{H}_{2}$.

4) Case Four: At last, we consider the circumstance that the relay can neither decode $X_{1}$ nor $X_{2}$. Thus, the relay remains silent in the second time slot, and the outage events at the destination are determined by the direct transmissions only.The outage probability is given by

$$
P_{o}^{4}=\operatorname{Pr}\left(\overline{\mathcal{E}}_{\left(\mathcal{S}_{1}, \mathcal{R}\right)} \bigcap \overline{\mathcal{E}}_{\left(\mathcal{S}_{2}, \mathcal{R}\right)}, \overline{\mathcal{E}}_{\mathcal{D}}\right),
$$

where $\operatorname{Pr}\left(\overline{\mathcal{E}}_{\left(\mathcal{S}_{1}, \mathcal{R}\right)} \cap \overline{\mathcal{E}}_{\left(\mathcal{S}_{2}, \mathcal{R}\right)}\right)=P_{o, 3} . \operatorname{Pr}\left(\overline{\mathcal{E}}_{\mathcal{D}}\right)$ are obtained similar to the outage probability at MAC-relay case. Combin- 


$$
\begin{aligned}
& I\left(x_{1}^{j}, x_{2}^{j} ; y_{\mathcal{R}}^{j}\right)=\frac{1}{4} \sum_{u=-1,1} \sum_{v=-1,1} \int_{-\infty}^{\infty} p\left(y_{\mathcal{R}}^{j} \mid x_{1}^{j}=u, x_{2}^{j}=v\right) \\
& \log \left(\frac{4 p\left(y_{\mathcal{R}}^{j} \mid x_{1}^{j}=u, x_{2}^{j}=v\right)}{p\left(y_{\mathcal{R}}^{j} \mid x_{1}^{j}=1, x_{2}^{j}=1\right)+p\left(y_{\mathcal{R}}^{j} \mid x_{1}^{j}=1, x_{2}^{j}=-1\right)+p\left(y_{\mathcal{R}}^{j} \mid x_{1}^{j}=-1, x_{2}^{j}=1\right)+p\left(y_{\mathcal{R}}^{j} \mid x_{1}^{j}=-1, x_{2}^{j}=-1\right)}\right) \mathrm{d} y .
\end{aligned}
$$

ing equations (10), (12), (14) and (15), we obtain the systemlevel overall outage probability as

$$
P_{o, s y s}=\sum_{i=1}^{4} P_{o}^{i} .
$$

\section{Ber AnAlysis Of ADAPtive DF Strategy}

In this section, we will analyze the bit error rate (BER) of the adaptive DF cooperation protocol in the multiple-access relay channels. We assume that when the channel is not in outage there still exists decoding error probability on the received signal. This is a practical assumption in real communication system. We first define the system BER equation assuming that if the decoding error occurs at the relay node, the decoding error at the destination is deterministic. This assumption is based on the fact that the source-relay channel gain is always stronger than the source-destination gain. Thus we have

$$
P_{e, s y s}=\sum_{i=1}^{4}\left\{P_{e_{i}}^{\mathcal{R}}+\left(1-P_{e_{i}}^{\mathcal{R}}\right) P_{e_{i}}^{\mathcal{D}}\right\},
$$

where $P_{e_{i}}^{\mathcal{R}}$ and $P_{e_{i}}^{\mathcal{D}}$ are the probabilities that an error happens at relay node and destination node in case $i$, respectively.

\section{A. BER Performance at Relay node}

In this subsection, we consider the BER performance corresponding to four outage cases at relay node, that is

- Case One: When the relay decodes both sources' messages correctly, and forwards $X_{\mathcal{R}}=X_{1} \oplus X_{2}$ to $\mathcal{D}$, the BER is shown in (18).

- Case Two: When the relay correctly decodes $X_{1}$, but not $X_{2}$, the BER is shown in (19).

- Case Three: When the relay correctly decodes $X_{2}$, but not $X_{1}$, the BER is shown in (20).

- Case Four: When the relay cannot decode neither $X_{1}$ nor $X_{2}$, the relay keeps silent in the second time slot, so $P_{e_{4}}^{\mathcal{R}}=0$,

where $Q(\cdot)$ is the Gaussian-Q function defined as $Q(x)=$ $\frac{1}{\sqrt{2 \pi}} \int_{x}^{\infty} \exp \left(-y^{2} / 2\right) d y$.

\section{B. BER Performance at Destination Node}

Now, we analyze the BER performance of the adaptive DF strategy at destination node. Combined with the received signal $Y_{1}$ from the direct link in the first time slot, the destination implement the following maximum likelihood decoding

$$
\left(\hat{x}_{1}^{j}, \hat{x}_{2}^{j}\right)=\arg \min _{x_{1}^{j}, x_{2}^{j}}\left|y_{1}^{j}-h_{1 \mathcal{D}} x_{1}^{j}-h_{2 \mathcal{D}} x_{2}^{j}\right|^{2}+\left|y_{2}^{j}-h_{\mathcal{R D}} x_{r}^{j}\right|^{2} .
$$

\begin{tabular}{|c|c|c|c|c|c|}
\hline$x_{1}^{j}$ & $x_{2}^{j}$ & $\left|y_{1}^{j}\right|$ & \multicolumn{4}{|c|}{$\left|y_{2}^{j}\right|$} \\
\cline { 4 - 6 } & +1 & $\left|h_{1 \mathcal{D}}+h_{2 \mathcal{D}}\right|$ & Case One & Case Two & Case Three \\
\hline+1 & -1 & $\left|h_{1 \mathcal{D}}-h_{2 \mathcal{D}}\right|$ & $\left|h_{\mathcal{R} \mathcal{D}}\right|$ & $-\left|h_{\mathcal{R} \mathcal{D} \mathcal{D}}\right|$ & $-\left|h_{\mathcal{R D}}\right|$ \\
\hline+1 & +1 & $-h_{1 \mathcal{D}}+h_{2 \mathcal{D}} \mid$ & $-\left|h_{\mathcal{R D}}\right|$ & $\left|h_{\mathcal{R} \mathcal{D}}\right|$ & $\left|h_{\mathcal{R} \mathcal{D}}\right|$ \\
\hline-1 & -1 & $-h_{1 \mathcal{D}}-h_{2 \mathcal{D}} \mid$ & $-\left|h_{\mathcal{R} \mathcal{D}}\right|$ & $-\left|h_{\mathcal{R} \mathcal{D}}\right|$ & $\left|h_{\mathcal{R} \mathcal{D}}\right|$ \\
\hline-1 & &
\end{tabular}

TABLE I

RELATIONSHIP BETWEEN THE REAL SOURCE TRANSMITTED SIGNALS AND THE RECEIVED SIGNALS WITHOUT NOISE CORRUPTION

To obtain the derivation of the BER, we view the direct link transmitted signals from two sources as a 4-PAM modulated signal, e.g., $x_{s}^{j} \triangleq x_{1}^{j}+x_{2}^{j}= \pm 1 \pm 1$. For coherent detection at the relay and destination nodes, the relationship between the real transmitted signals from source nodes and the received signals without noise corruption at $\mathcal{R}$ and $\mathcal{D}$ in Table IV-B. Based on these relationship, we construct a coordinate with received signal $y_{1}^{j}$ as the X-label and $y_{2}^{j}$ as the Y-label. According to relative absolute values of $h_{1 \mathcal{D}}$ and $h_{2 \mathcal{D}}$, the absolute value of $h_{\mathcal{R} \mathcal{D}}$, and the sign of $x_{1}^{j}$ and $x_{2}^{j}$, there exists 8 possible positions of reference points $\mathbb{M}_{i}=\left(\mathbb{x}_{i}, \mathbb{y}_{i}\right)$ with $\pm\left|h_{1 \mathcal{D}}\right| \pm\left|h_{2 \mathcal{D}}\right|$ as X-label and $\pm\left|h_{\mathcal{R D}}\right|$ as Y-label, for $i=1,2,3,4$. Without loss of generality, we assume that the direct link $\mathcal{S}_{1}-\mathcal{D}$ is poorer than link $\mathcal{S}_{2}-\mathcal{D}$, then the total number of possible positions of reference points reduces to 4. For illustration simplicity, we only introduce the cases that $\left|y_{2}^{i}\right|+\left|h_{\mathcal{R D}}\right|$ in detail, e.g., $x_{1}^{j}$ and $x_{2}^{j}$ sharing the same sign in Case one, $x_{1}^{j}=1$ in Case two, and $x_{2}^{j}=1$ in Case three, and give the final results of other 3 cases.

1) Case One: When the relay decodes both sources' messages correctly, and the destination receives $X_{\mathcal{R}}=X_{1} \oplus X_{2}$ in the second time slot, the coordinate is given by Fig. 3(a), where the lines $l_{1}$ and $l_{2}$ are vertical to the two sides of isosceles trapezoid, and the decoding areas are denoted by $\Omega_{i}$, for $i=1,2,3,4$. Specifically, the functions of $l_{1}$ and $l_{2}$ are given by $y_{2}^{j}=\frac{\left|h_{1 \mathcal{D}}\right|}{\left|h_{\mathcal{R} D}\right|} y_{1}^{j}-\frac{\left|h_{1 \mathcal{D}}\right|\left|h_{2 \mathcal{D}}\right|}{\left|h_{\mathcal{R D}}\right|}$ and $y_{2}^{j}=-\frac{h_{1 \mathcal{D}}}{h_{\mathcal{R} \mathcal{D}}} y_{1}^{j}-\frac{\left|h_{1 \mathcal{D}}\right|\left|h_{2 \mathcal{D}}\right|}{\left|h_{\mathcal{R} \mathcal{D}}\right|}$, respectively. And the coordination of center of the circle is $\left(0,-\frac{\left|h_{1 \mathcal{D}}\right|\left|h_{2 \mathcal{D}}\right|}{\left|h_{\mathcal{R} \mathcal{D}}\right|}\right)$. Besides, $\Omega_{1}$ is the area that $\left\{l_{1} \leq 0 \cup y_{2}^{j} \leq-\frac{\left|h_{1 \mathcal{D}}\right|\left|h_{2 \mathcal{D}}\right|}{\left|h_{\mathcal{R} \mathcal{D}}\right|}\right\} ; \Omega_{2}$ is the area that $\left\{l_{1}>0 \cup y_{2}^{j}>-\frac{\left|h_{1 \mathcal{D}}\right|\left|h_{2 \mathcal{D}}\right|}{\left|h_{\mathcal{R} \mathcal{D}}\right|}\right\} ; \Omega_{3}$ is the area that $\left\{l_{2}>0 \cup y_{2}^{j}>-\frac{\left|h_{1 \mathcal{D}}\right|\left|h_{2 \mathcal{D}}\right|}{\left|h_{\mathcal{R} D}\right|}\right\}$; and $\Omega_{4}$ is the area that $\left\{l_{2} \leq 0 \cup y_{2}^{j} \leq-\frac{\left|h_{1 \mathcal{D}}\right|\left|h_{2 \mathcal{D}}\right|}{\left|h_{\mathcal{R} \mathcal{D}}\right|}\right\}$. Since the noise samples $N_{1}^{j}$ and $N_{2}^{j}$ are independently complex Gaussian distributed random variables with zero-mean and variance $\sigma^{2}$ and we assume 


$$
P_{e_{1}}^{\mathcal{R}}=\left\{\begin{array}{l}
Q\left(\frac{\left|h_{2 \mathcal{R}}\right|}{\sigma}\right)+\frac{1}{2}\left[Q\left(\frac{2\left|h_{1 \mathcal{R}}\right|-\left|h_{2 \mathcal{R}}\right|}{\sigma}\right)+Q\left(\frac{2\left|h_{1 \mathcal{R}}\right|+\left|h_{2 \mathcal{R}}\right|}{\sigma}\right)\right] \\
Q\left(\frac{\left|h_{1 \mathcal{R}}\right|}{\sigma}\right)+\frac{1}{2}\left[Q\left(\frac{2\left|h_{2 \mathcal{R}}\right|-\left|h_{1 \mathcal{R}}\right|}{\sigma}\right)+Q\left(\frac{2\left|h_{2 \mathcal{R}}\right|+\left|h_{1 \mathcal{R}}\right|}{\sigma}\right)\right] \\
\left|h_{1 \mathcal{R}}\right|>\left|h_{2 \mathcal{R}}\right| \\
\left|h_{1 \mathcal{R}}\right| \leq\left|h_{2 \mathcal{R}}\right|
\end{array} ;\right.
$$

$$
P_{e_{2}}^{\mathcal{R}}=\left\{\begin{array}{c}
\frac{1}{2}\left[Q\left(\frac{\left|h_{1 \mathcal{R}}\right|+\left|h_{2 \mathcal{R}}\right|}{\sigma}\right)+Q\left(\frac{\left|h_{1 \mathcal{R}}\right|-\left|h_{2 \mathcal{R}}\right|}{\sigma}\right)\right]\left|h_{1 \mathcal{R}}\right|>\left|h_{2 \mathcal{R}}\right| \\
Q\left(\frac{\left|h_{1 \mathcal{R}}\right|}{\sigma}\right)+\frac{1}{2}\left[Q\left(\frac{2\left|h_{2 \mathcal{R}}\right|+\left|h_{1 \mathcal{R}}\right|}{\sigma}\right)+Q\left(\frac{\left|h_{2 \mathcal{R}}\right|-\left|h_{1 \mathcal{R}}\right|}{\sigma}\right)-Q\left(\frac{\left|h_{1 \mathcal{R}}\right|+\left|h_{2 \mathcal{R}}\right|}{\sigma}\right)-Q\left(\frac{2\left|h_{2 \mathcal{R}}\right|-\left|h_{1 \mathcal{R}}\right|}{\sigma}\right)\right]\left|h_{1 \mathcal{R}}\right| \leq\left|h_{2 \mathcal{R}}\right|
\end{array} ;\right.
$$

$P_{e_{3}}^{\mathcal{R}}=\left\{\begin{array}{c}Q\left(\frac{\left|h_{2 \mathcal{R}}\right|}{\sigma}\right)++\frac{1}{2}\left[Q\left(\frac{2\left|h_{\mathcal{R}}\right|+\left|h_{2 \mathcal{R}}\right|}{\sigma}\right)+Q\left(\frac{\left|h_{1 \mathcal{R}}\right|-\left|h_{2 \mathcal{R}}\right|}{\sigma}\right)-Q\left(\frac{\left|h_{2 \mathcal{R}}\right|+\left|h_{1 \mathcal{R}}\right|}{\sigma}\right)-Q\left(\frac{2\left|h_{1 \mathcal{R}}\right|-\left|h_{2 \mathcal{R}}\right|}{\sigma}\right)\right]\left|h_{1 \mathcal{R}}\right| \leq\left|h_{2 \mathcal{R}}\right| \\ \frac{1}{2}\left[Q\left(\frac{\left|h_{1 \mathcal{R}}\right|+\left|h_{2 \mathcal{R}}\right|}{\sigma}\right)+Q\left(\frac{\left|h_{2 \mathcal{R}}\right|-\left|h_{1 \mathcal{R}}\right|}{\sigma}\right)\right]\left|h_{1 \mathcal{R}}\right|>\left|h_{2 \mathcal{R}}\right|\end{array} ;\right.$
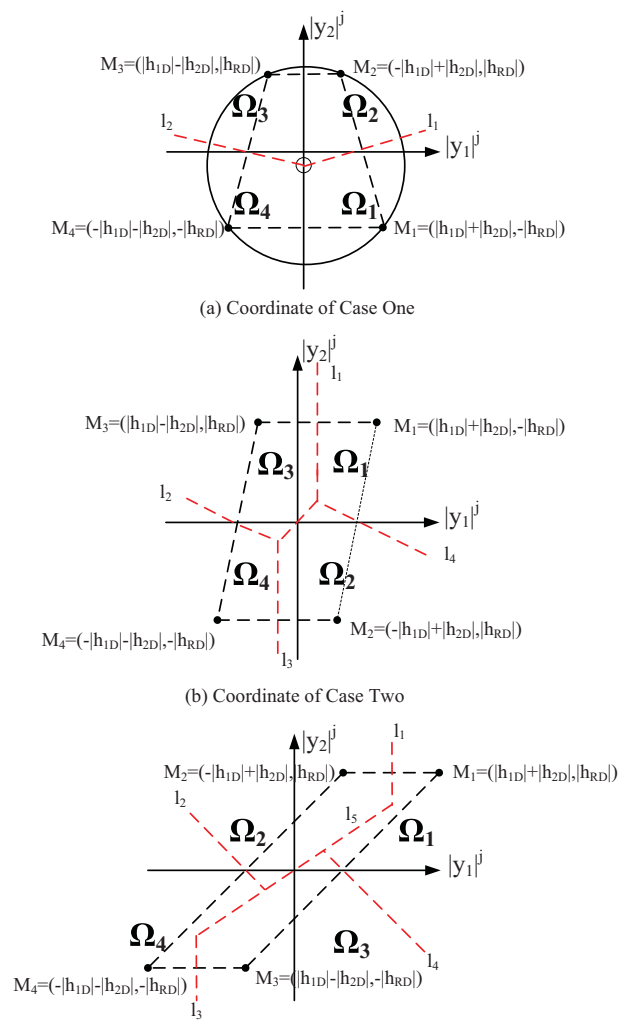

(c) Coordinate of Case Three

Fig. 3. Coordinate of Three Cases

the system is perfectly synchronized, the error probability of Case one is given by

$$
P_{e_{1}}^{\mathcal{D}}=\sum_{i=1}^{4}\left\{1-\iint_{\Omega_{i}} \mathcal{N}\left(\left|y_{1}^{j}\right|,\left|y_{2}^{j}\right| ; \mathbb{x}_{i}, \mathbb{y}_{i}, \sigma^{2}\right) d \Omega_{i}\right\},
$$

2) Case Two: When the relay is only able to decode $X_{1}$ correctly, and the destination receives $X_{\mathcal{R}}=X_{1}$ in the second time slot, the coordinate is given by Fig. 3(b), where the lines $l_{k}$ for $k=1, \cdots, 4$ are vertical to the four edges of the parallelogram, $l_{5}$ is the diagonal of the parallelogram, and the decoding areas are denoted by $\Omega_{i}$, for $i=1,2,3,4$. Specifically, the function of $l_{1}$ is given by $y_{1}^{j}=\left|h_{1 \mathcal{D}}\right|$; the function of $l_{2}$ is $y_{2}^{j}=-\frac{\left|h_{1 \mathcal{D}}\right|}{\left|h_{\mathcal{R} D}\right|} y_{1}^{j}-\frac{\left|h_{\mathcal{D} D}\right|\left|h_{2 \mathcal{D}}\right|}{\left|h_{\mathcal{R} D}\right|}$; the function of $l_{3}$ is $y_{1}^{j}=-\left|h_{1 \mathcal{D}}\right|$; the function of $l_{4}$ is $y_{2}^{j}=-\frac{\left|h_{1 \mathcal{D}}\right|}{\left|h_{\mathcal{R} \mathcal{D}}\right|} y_{1}^{j}+\frac{\left|h_{1 \mathcal{D}}\right|\left|h_{2 \mathcal{D}}\right|}{\left|h_{\mathcal{R} D}\right|}$; the function of $l_{5}$ is $y_{2}^{j}=$ $\frac{\left|h_{\mathcal{R} D}\right|}{\left|h_{1 \mathcal{D}}\right|+\left|h_{2}\right|} y_{1}^{j}$. And the decoding spaces are the surrounded areas defined by these lines, that is $\Omega_{1}=\left\{l_{1}>0 \cup l_{4}>0\right\}$; $\Omega_{2}=\left\{l_{3}>0 \cup l_{4} \leq 0 \cup l_{5} \leq 0\right\} ; \Omega_{3}=\left\{l_{2} \leq 0 \cup l_{3} \leq 0\right\} ;$ and $\Omega_{2}=\left\{l_{1} \leq 0 \cup l_{2}>0 \cup l_{5}>0\right\}$. Thus, the error probability of Case two is determined by equation (22) with the defined decoding areas of case two.

3) Case Three: When the relay is only able to decode $X_{2}$ correctly, and the destination receives $X_{\mathcal{R}}=X_{2}$ in the second time slot, the coordinate is given by Fig. 3(c), where the lines $l_{k}$ for $k=1, \cdots, 4$ are vertical to the four edges of the parallelogram, $l_{5}$ is the diagonal of the parallelogram, and the decoding areas are denoted by $\Omega_{i}$, for $i=1,2,3,4$. Specifically, the function of $l_{1}$ is given by $y_{1}^{j}=\left|h_{2 \mathcal{D}}\right|$; the function of $l_{2}$ is $y_{2}^{j}=-\frac{\left|h_{2 \mathcal{D}}\right|}{\left|h_{\mathcal{R} D}\right|} y_{1}^{j}-\frac{\left|h_{1 \mathcal{D}}\right|\left|h_{2 \mathcal{D}}\right|}{\left|h_{\mathcal{R} D}\right|}$; the function of $l_{3}$ is $y_{1}^{j}=-\left|h_{2 \mathcal{D}}\right|$; the function of $l_{4}$ is $y_{2}^{j}=-\frac{\left|h_{2 \mathcal{D}}\right|}{\left|h_{\mathcal{R} D}\right|} y_{1}^{j}+$ $\frac{\left|h_{1 \mathcal{D}}\right|\left|h_{2 \mathcal{D}}\right|}{\left|h_{\mathcal{R} D}\right|}$; and the function of $l_{5}$ is $y_{2}^{j}=\frac{\left|h_{\mathcal{R} D}\right|}{\left|h_{1 \mathcal{D}}\right|+\left|h_{2 \mathcal{D}}\right|} y_{1}^{j}$. And the decoding spaces are the surrounded areas defined by these lines, that is $\Omega_{1}=\left\{l_{1}>0 \cup l_{4}>0 \cup l_{5} \leq 0\right\}, \Omega_{2}=\left\{l_{1} \leq\right.$ $\left.0 \cup l_{2}>0 \cup l_{5}>0\right\}, \Omega_{3}=\left\{l_{3}>0 \cup l_{4} \leq 0 \cup l_{5} \leq 0\right\}$, and $\Omega_{4}=\left\{l_{2} \leq 0 \cup l_{3} \leq 0 \cup l_{5}>0\right\}$. Thus, the error probability of Case three is determined by equation (22) with the defined decoding areas of case three.

4) Case Four: When the relay cannot decode neither $X_{1}$ nor $X_{2}$, the destination decodes the sources' messages by using the information from the direct links only. Then we implement the error probability 4-PAM modulated signal [10], 


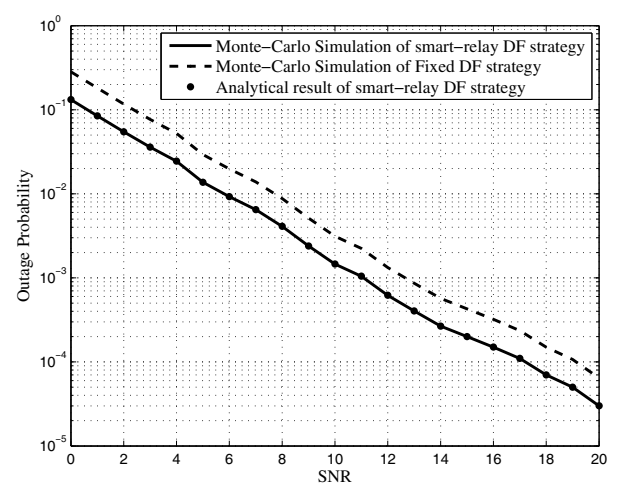

Fig. 4. Outage probability of adaptive DF strategy and fixed DF strategy.

that is

$$
P_{e_{4}}^{\mathcal{D}}=\frac{2(M-1)}{M} \cdot Q\left(\sqrt{\frac{6 \log _{2} M \rho}{M^{2}-1}}\right),
$$

where $M=4$ in this case, and $\rho$ denotes the instantaneous SNR, which equals to $\mathbb{E}\left\{\left|h_{1 \mathcal{D}}\right|^{2}\left|X_{1}\right|^{2}+\left|h_{2 \mathcal{D}}\right|^{2}\left|X_{2}\right|^{2}\right\} / \sigma^{2}$.

\section{Simulation Results}

We assume both sources have the same power, have the same rate, and share the same distance to the relay and destination nodes. We further assume that $\varphi_{1}=\varphi_{2}=\pi$. The distance between each source and the relay is $d_{\mathcal{R}}=0.5$; the distance between the relay and the destination is $d_{\mathcal{R} \mathcal{D}}=0.5$; and the distance between $\mathcal{S}_{i}$ and the destination is $d_{i \mathcal{D}}=1$. The channel attenuation exponents is $\gamma=2$. The signal-tonoise ratio (SNR) in all the simulations is defined as the transmission SNR of each source, i.e., $\frac{1}{\sigma^{2}}$.

Fig. 4 shows the outage probability of adaptive DF strategy based on theoretical analysis and on Monte-Carlo simulation, respectively, and compares to the outage probability of the fixed DF strategy from $0 \mathrm{~dB}$ to $20 \mathrm{~dB}$. Here, the fixed strategy states that the relay transmits the network-coded signal at all the time without considering the outage events of source-relay channels. The derived outage probability expression is shown to be in tight match with Monte-Carlo simulation. The diversity order of the proposed scheme is two. And the adaptive DF strategy has a better outage probability performance than the fixed DF strategy.

Fig. 5 shows the BER performance of adaptive DF strategy compared with the fixed DF strategy from $0 \mathrm{~dB}$ to $10 \mathrm{~dB}$. The analytical BER result is shown to be in tight match with Monte-Carlo simulation. And the adaptive DF strategy has a better BER performance than the fixed DF strategy.

\section{Vi. Conclusion}

In this paper, we propose an adaptive decode-and-forward strategy in multiple-access relay channels. The outage probability of the adaptive DF strategy is analyzed. Later we propose a BER calculation method based on the efficient coordinates. The outage probability of the adaptive DF strategy, and system

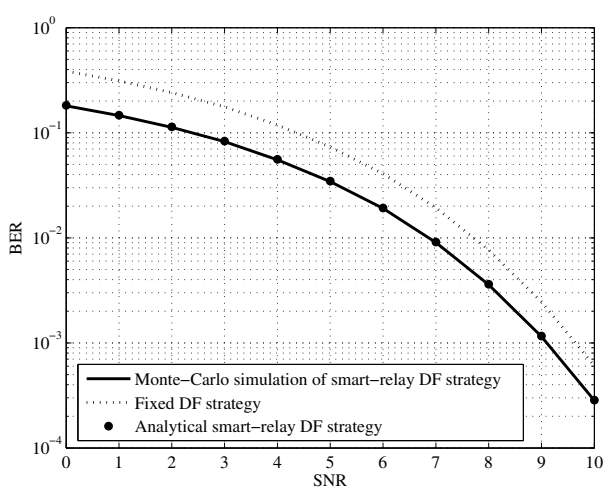

Fig. 5. BER performance of adaptive DF strategy and fixed DF strategy.

BER expressions are shown to be in tight match with MonteCarlo simulation results. Simulation results reveal that adaptive DF strategy yields SNR gains compared to the fixed DF strategy.

\section{ACKNOWLEDGEMENT}

This work is supported by NSFC \#60972031, by national 973 project \#2012CB316106 and \#2009CB824900, by NSFC \#61161130529, by national huge special project \#2012ZX03004004, by national key laboratory project \#ISN11-01, by Huawei Funding \#YBWL2010KJ013.

\section{REFERENCES}

[1] Chen Gong; Guosen Yue; Xiaodong Wang; , "Joint Channel and Network Code Design for Half-Duplex Multiple-Access Relay System," Соттиnications (ICC), 2010 IEEE International Conference on, vol., no., pp. 15, 23-27 May 2010.

[2] Zeitler, G.; Koetter, R.; Bauch, G.; Widmer, J.; , "On Quantizer Design for Soft Values in the Multiple-Access Relay Channel," Communications, 2009. ICC 'O9. IEEE International Conference on, vol., no., pp.1-5, 14 18 June 2009.

[3] Woradit, K.; Quek, T.Q.S.; Suwansantisuk, W.; Win, M.Z.; Wuttisittikulkij, L.; Wymeersch, H.; , "Outage behavior of selective relaying schemes," Wireless Communications, IEEE Transactions on, vol. 8, no. 8 , pp. 3890-3895, August 2009.

[4] Farhadi, G.; Beaulieu, N.C.; , "On the Outage and Error Probability of Amplify-And-Forward Multi-Hop Diversity Transmission Systems,' Communications, 2008. ICC '08. IEEE International Conference on, vol. no., pp. 3748-3754, 19-23 May 2008.

[5] Ikki, S.S.; Ahmed, M.H.; "Performance analysis of adaptive decodeand-forward cooperative diversity networks with best-relay selection,' Communications, IEEE Transactions on, vol. 58, no. 1, pp. 68-72, January 2010.

[6] Woldegebreal, D.H.; Karl, H.; , "Multiple-Access Relay Channel with Network Coding and Non-Ideal Source-Relay Channels," Wireless Communication Systems, 2007. ISWCS 2007. 4th International Symposium on, vol., no., pp.732-736, 17-19 Oct. 2007.

[7] R. Narasimhan, "Individual outage rate regions for fading multiple access channels," in Proc. IEEE International Symposium on Information Theory (ISIT), 2007, pp. 1571-1575.

[8] Y. Ko; Alouini, M.-S.; Simon, M.K.; , "Outage probability of diversity systems over generalized fading channels," Communications, IEEE Transactions on, vol. 48, no. 11, pp. 1783-1787, Nov 2000.

[9] D. N. C. Tse, P. Viswanath, and L. Zheng, "Diversity-multiplexing tradeoff in multiple-access channels," IEEE Trans. Inform. Theory, vol. 50, no. 9, pp. 1859-1874, 2004.

[10] M. K. Simon and M. S. Alouini, Digital Communication Over Fading Channels: A Unified Approach to Performance Analysis. New York: Wiley, 2000. 